\title{
Foreword
}

\section{The Evolution of Antithrombotic Care in Cardiovascular Patients}

\author{
A. John Camm ${ }^{1}$ \\ ${ }^{1}$ St George's University of London and Imperial College, London, \\ United Kingdom
}

Thromb Haemost 2018;118 (Suppl 1):S1.

The direct, oral, factor Xa inhibitor rivaroxaban has been used in clinics for many years. The knowledge garnered from this nonvitamin $\mathrm{K}$ antagonist (VKA) oral anticoagulant (NOAC) has continued to grow since its first approval in 2008 for the prevention of venous thromboembolism (VTE) in patients undergoing elective hip or knee replacement surgery and subsequent approvals for prevention of stroke and systemic embolism in adult patients with nonvalvular atrial fibrillation (NVAF), treatment and prevention of recurrent VTE and the prevention of atherothrombotic events in adult patients following an acute coronary syndrome (ACS). Through an extensive research program, Bayer AG and Janssen Research \& Development, LLC strive to identify-and subsequently answer-important clinical questions that arise in thrombosis research, and the potential benefits of rivaroxaban in wider patient populations.

It is through landmark randomized controlled trials (RCTs) such as the Phase III Cardiovascular OutcoMes for People using Anticoagulation StrategieS (COMPASS) trial that we have learned important lessons around the benefits of a 'vascular' dose of rivaroxaban (2.5 $\mathrm{mg}$ twice daily [bid]) in combination with acetylsalicylic acid (ASA)-supporting the concept of combined antithrombotic therapy with an anticoagulant and an antiplatelet agent, to provide long-lasting vascular protection. In the setting of secondary stroke prevention, the Phase III oPen-label, randomized, controlled, multicentre study explorIng twO treatmeNt stratEgiEs of Rivaroxaban and a doseadjusted oral vitamin $\mathrm{K}$ antagonist treatment strategy in patients with Atrial Fibrillation who undergo Percutaneous Coronary Intervention (PIONEER AF-PCI) trial is the first to show benefits of a NOAC (rivaroxaban $15 \mathrm{mg}$ once daily [od]) + a P2Y 12 inhibitor, by presenting a lower rate of clinically significant bleeding versus a VKA + dual antiplatelet therapy, in patients with NVAF who underwent PCI with stent placement. Moreover, this regimen has recently been approved by the European Medicines Agency (EMA) for use in the aforementioned population for a maximum of 12 months' duration. We have seen further novel data from the Phase III EINSTEIN CHOICE trial, which concluded that rivaroxaban $10 \mathrm{mg}$ od, when used for extended VTE treatment, reduced the risk of recurrent VTE without significantly increasing the risk of

Address for correspondence

Professor A. John Camm,

St George's University of London

and Imperial College, London,

United Kingdom

(e-mail: jcamm@sgul.ac.uk). bleeding compared with ASA (following 6-12 months of anticoagulation). These data have subsequently led to label updates by both the EMA and the Food and Drug Administration to include extended prevention of recurrent VTE with rivaroxaban $10 \mathrm{mg}$ od following $\geq 6$ months of standard anticoagulation therapy. EINSTEIN CHOICE data suggest this alternative lower rivaroxaban dose may be a potentially valuable option for those whose benefit-risk profile requires less intense treatment, without a compromise on clinical benefit. These and other RCTs are summarized in the following series of articles.

Real-world data are also in abundance and allow further characterization of unmet medical needs in the management of thrombosis. Such evaluations offer important opportunities to generate robust data complementary to those from RCTs. Considerations around generation of high-quality realworld data will be covered in this series, along with updates from several real-world analyses focusing on rivaroxaban for stroke prevention in AF. These include a pooled analysis that was performed for three prospective, observational studies in the XArelto on preveNtion of sTroke and non-central nervoUS system systemic embolism in patients with nonvalvular atrial fibrillation (XANTUS) program, which assessed the global safety profile of rivaroxaban in routine practice, collecting information on AF patients in 47 countries from Western and Eastern Europe, Canada and Israel; Asia-Pacific and Eastern Europe; and the Middle East, Africa and Latin America. In addition, we appraise findings from a selection of country-specific datasets, including the Danish cohort study, the REAFFIRM study (US) and the U.S. Department of Defense Military Health Service Post-Marketing Safety Surveillance study. Even while evaluating global patient populations using a wide variety of study designs, we continue to see consistent benefits for rivaroxaban in $\mathrm{AF}$ patients in routine clinical practice.

I anticipate that these articles will provide an interesting perspective on the comprehensive rivaroxaban research program, which is continuously identifying novel rivaroxaban treatment strategies in stroke prevention, vascular protection and venous protection.

Copyright () 2018 Schattauer

DOI https://doi.org/ 10.1055/s-0038-1626720. ISSN 0340-6245. 